\title{
An efficient two-step method to purify very small embry- onic-like (VSEL) stem cells from umbilical cord blood (UCB)
}

\author{
Maciej Halasa, Magdalena Baskiewicz-Masiuk, Elżbieta Dabkowska, \\ Bogusław Machalinski
}

Department of General Pathology, Pomeranian Medical University, Szczecin, Poland

\begin{abstract}
The identification in murine bone marrow (BM) of very small embryonic-like (VSEL) stem cells, possessing several features of pluripotent stem cells, encouraged us to investigate if similar population of cells could be also isolated from the human umbilical cord blood (UCB). Here our approach to purify VSEL from human UCB is described by employing a two step isolation strategy based on i) hypotonic lysis of erythrocytes followed ii) by multi-parameter FACS sorting. Accordingly, first, erythrocytes are removed from the UCB samples by hypotonic ammonium chloride solution and next, the UCB mononuclear cells (UCB MNC) are stained with monoclonal antibodies against all hematopoietic lineages including the common leukocyte antigen CD45. The cells carrying these markers $\left(\operatorname{lin}^{+} \mathrm{CD} 45^{+}\right)$are eliminated from the sort by electronic gating. At the same time the antibodies against CXCR4, CD34 and CD133 are employed as positive markers to enrich the UCB MNC for VSEL. This combined two step approach enables to purify VSEL stem cells, which are small and express mRNA for pluripotent stem cells (PSC) (Oct-4 and Nanog) and tissue-committed stem cells (TCSC) (Nkx2.5/Csx, VE-cadherin and GFAP) similarly to those isolated from the adult BM (3-5 $\mu \mathrm{m}$ cells with large nuclei).
\end{abstract}

Key words: VSEL - Cord blood - Multiparameter sorting - CXCR4

\section{Introduction}

Search for the source of the pluripotent stem cells (PSC) capable of prducing the tissues of various kinds has been seriously withheld when it became apparent that the only known efficient source of such cells in humans can be found in the embryos. This has clearly set the ethical barrier, which delayed further studies on the PSC, as well as aroused serious concerns for potential use of these cells in medical practice.

Until know the available evidence suggested that the cells possessing pluripotent potential, able to regenerate various tissues, could be found in nonembryonic tissues like the BM or UCB [1-7]. Recently a population of the very small embryonic-like (VSEL) stem cells was isolated from the adult murine BM [8] as well as from the other adult tissues [9-10].

Correspondence: M. Halasa, B. Machaliński, Dept. of General Pathology, Pomeranian Medical University, Al. Powstancow Wlkp. 72, 70-111 Szczecin, Poland; tel.: (+4891) 4661546, fax.: (+4891) 4661548, e-mail: maciupam@sci.pam.szczecin.pl or machalin@sci.pam.szczecin.pl
VSEL were sorted from the BM as a population of small Sca- $1^{+}$lin ${ }^{-C D} 45^{-}$cells that express also CXCR4 receptor [8]. It was confirmed, that these cells express markers characteristic of embryonic PSC, like SSEA1, Oct-4, Nanog and Rex-1, as well as they differentiate in vitro into cells from all three germ layers [8]. This discovery gave a new impulse to the researches in adult stem cells and their potential application in regenerative medicine [11].

The identification of VSEL in mice prompted us to search for a similar population of stem cells in humans. Since UCB had been described as a potential source of primitive stem cells [12-14], we employed an analogical strategy as for the murine BM to sort these cells from human UCB. Based on our experience with purifying VSEL from murine tissues, we assumed that similarly as in mice, human VSEL will be also very small in size [8].

Using the technique based on the novel two-step procedure $\left(1^{\text {st }}\right.$ step - removal of the erythrocytes from the sample by hypotonic ammonium chloride lysis, and the $2^{\text {nd }}$ step - multiparameter high speed sorting) we successfully isolated VSEL stem cells from the 


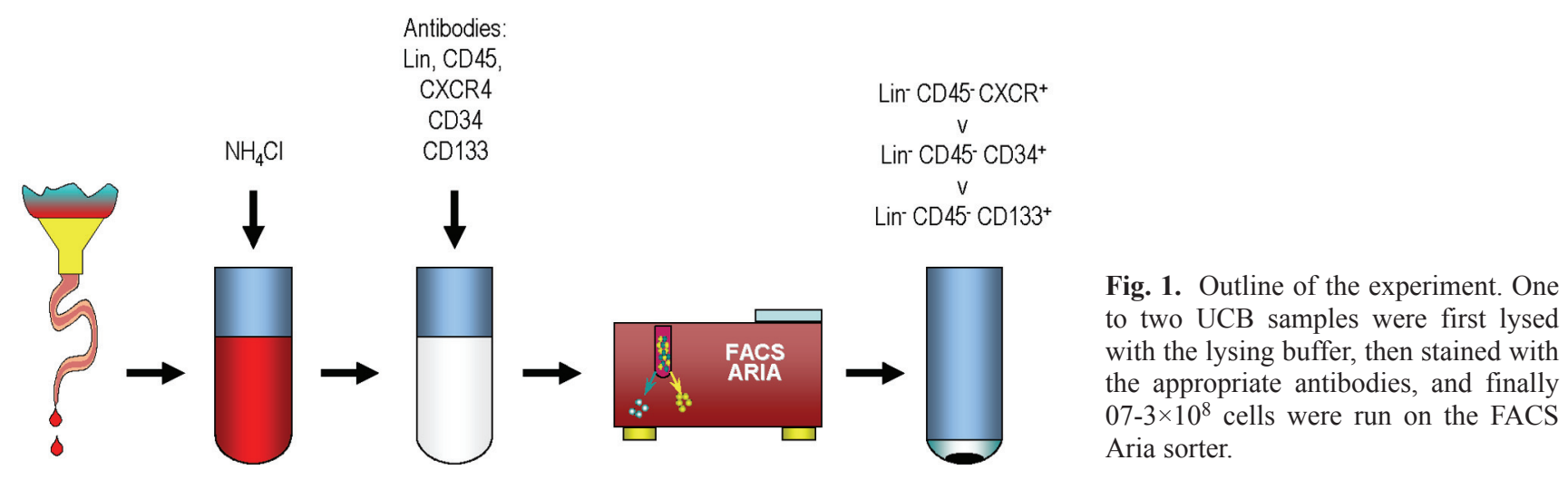

UCB. Here this procedure is described in details with the possible technical problems that have to be solved for successful VSEL stem cell isolation.

\section{Materials and methods}

UCB collection. The UCB was obtained from the umbilical cords and placenta after the delivery of placenta and maximum attention has been paid to aseptic procedures. The UCB was harvested to the containers and mixed with anticoagulant (heparin - 2500 I.U. per $\mathrm{ml}$ of UCB). The usual amount of blood harvested from one umbilical cord ranged between 10 and $60 \mathrm{ml}$. The blood was then stored at $4^{\circ} \mathrm{C}$ for up to two days before further processing. To increase the volume of the sorted UCB, usually two independently obtained samples were combined.

Hypotonic removal of erythrocytes. The UCB was then mixed with $\mathrm{NH}_{4} \mathrm{Cl}$ based BD Pharm Lyse lysing buffer (BD Biosciences, San Jose, CA, USA) for $15 \mathrm{~min}$ at room temperature and washed twice in phosphate-buffered saline (PBS). This step was repeated up to three times, depending on the efficiency of lysis.

FACS-based sorting of VSEL. Next, $1-3 \times 10^{8}$ of UCB MNC were stained with monoclonal antibodies. For linage markers we used antibodies against: CD2 clone RPA-2.10, CD3 clone SK7, CD14 clone $\mathrm{M} \varphi \mathrm{P} 9, \mathrm{CD} 16$ clone 3G8, CD19 clone HIB19, CD24 clone ML5, CD56 clone NCAM16.2, CD66b clone G10F5, CD235a clone GA-R2. All the linage markers directed antibodies were conjugated with fluorescein isothiocyanate (FITC). The anti-CD45 (clone $\mathrm{H} 130)$ was conjugated with phycoerythrin (PE), and antibodies against CXCR4 (clone 12G5), CD34 (clone 581) and CD133 (clone CD133/1) (Miltenyi Biotec GmbH) were all conjugated with allophycocyanin (APC). The antibodies were provided by BD Pharmingen, San Jose, CA, USA, except otherwise specified. The samples were incubated with antibodies at room temperature for $30 \mathrm{~min}$. After being washed, the cells were run through the $40 \mu \mathrm{m}$ gauge filters (BD Falcon, San Jose, CA, USA) to remove potential clots. The final sample concentration was $2-8 \times 107$ cells $/ \mathrm{ml}$ of PBS.

The sort was performed on the FACSAria instrument (BD, San Jose, CA, USA) utilizing the high speed sorting settings with the use of $70 \mu \mathrm{m}$ nozzle. The basic principles of gating for sort the were: i) the P1 gate on the SSC/FSC dot plot was set on the lymphocyte region with the inclusion of very small cells, ii) the P1 restricted P2 gate on the FITC/APC dot plot was set the way that only non-lineage markers expressing cells and the APC conjugated antibodies positive cells $\left(\mathrm{CXCR} 4^{+}\right.$or $\mathrm{CD} 34^{+}$or $\left.\mathrm{CD} 133^{+}\right)$were included, iii) the $\mathrm{P} 2$ restricted $\mathrm{P} 3$ gate on the $\mathrm{SSC} / \mathrm{PE}$ dot plot was set to include only the CD45 negative cells. The cells were collected to the tube coated with $10 \%$ FBS.
General outline of the experiment is presented in Fig. 1. The data presented in the paper are based on the sorts performed on 28 UCB samples.

\section{Results and discussion}

By employing FACS analysis it was found that cells expressing the potential VSEL phenotype $\left(\mathrm{CXCR} 4^{+}\right.$ lin-CD45-, CD $34^{+}{ }^{-}$lin $^{-} \mathrm{CD} 45^{-}$and $\left.\mathrm{CD} 133^{+}{ }^{-} \mathrm{in}^{-} \mathrm{CD} 45^{-}\right)$ are very rare and could be found in the human UCB with the following rates respectively: $0037 \% \pm 0.02$ $\mathrm{n}=9,0.118 \% \pm 0.028 \mathrm{n}=5$ and $0.018 \% \pm \mathrm{n}=9$ (9). Thus, based on a very low frequency of these cells in order to isolate them from UCB we decided to avoid immunomagnetic beads isolation as a potentially less efficient method and employed FACS-based multiparameter sorting.

However, the choice of high speed FACS sorting as an isolation method of VSEL required elimination of the erythrocytes from the samples before UCB MNC could be run on the sorter. Since the removal of red blood cells (RBC) by centrifugation over Ficoll-Paque gradient might lead to the loss of the VSEL stem cells from UCB samples due to their very small size and possibility of being engulfed by sedimenting RBCs, we employed hypotonic ammonium chloride solution to lyse RBC. When necessary, this lysis procedure had to be repeated up to two-three times.

The removal of erythrocytes from the UCB samples prepared for sorting turned out to be crucial, as their presence in the P1 gate on SSC/FSC dot plot overlapped with the cytogram of the VSEL stem cells which are located close to the lymphocyte region (Fig. 2). As it is shown in Fig. 2 because of the small cell size of the VSEL we had to move the sorting gate to the left to the area that contains RBCs and cell debris.

Fig. 3 shows a dot plot containing cells from the P1 gate, which was set to include cells that are hematopoietic lineages negative (all anti-lineage antibodies were conjugated with FITC), and express either CXCR4 or CD34 or CD133 antigens. In several experiments we alternatively stained aliquots of the same sample indi- 


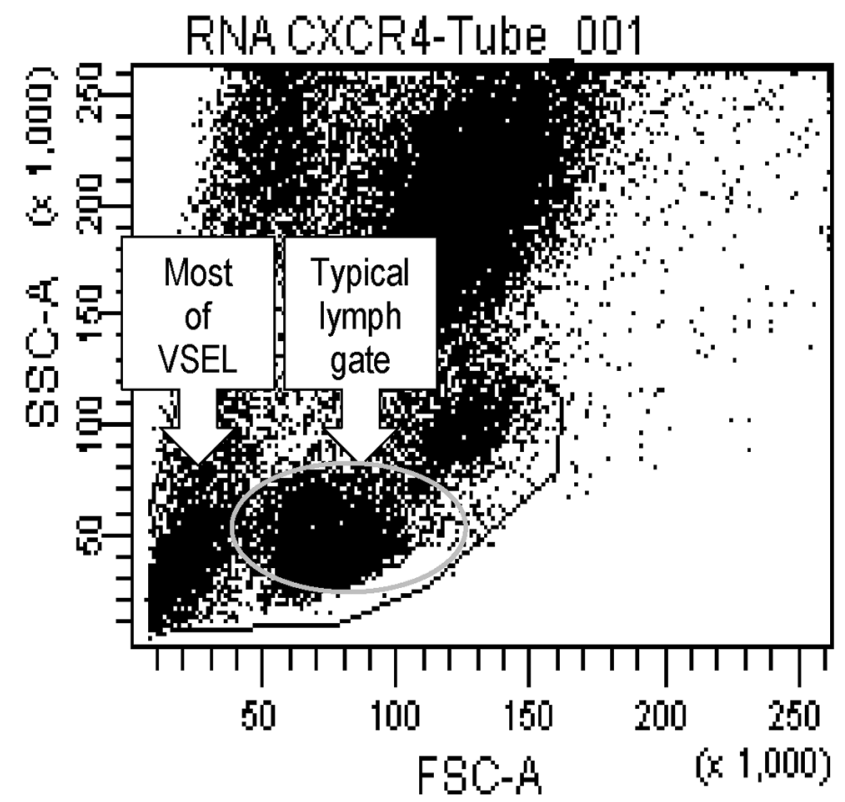

Fig. 2. Gating on the SSC/FSC dot plot was one of the most important steps in the sort setup. The threshold set on FSC has to be set very low, even despite some RBCs remnants/debris obstructing the view. The VSEL on this plot are located far from the main population of lymphocytes, thus the P1 gate has to be extended to the left towards small events.

vidually with each of these three markers and were able to isolate VSEL, although in some of these attempts the CD133 use has given markedly smaller number of sorted cells from the same sample.

Fig. 4 shows a dot plot, where the CD45 expression was analyzed against the SSC, to gate the CD45 negative cells from those present in the gates P1 and P2. This step was crucial to exclude potential hematopoietic stem cells from the sorted cell population.

There were certain issues, however, that we had to consider in setting up our sorting strategy. First, it was important to assess how restrictive the gating should be in order to allow for high enrichment of VSEL. When the subpopulations of the cells abundantly represented in the sample are sorted, the settings of the sort can be as restrictive as it is needed, to harvest the highly purified population of cells. However, our goal to sort the relatively rare cells such as lin-CD45$\mathrm{CXCR}^{+}$, lin-CD45-CD34 ${ }^{+}$or lin-CD45-CD $133^{+}$ UCB MNC, which could be found in the UCB samples with frequencies between approximately 1 in $1 \times 10^{4}$. $1 \times 10^{5}$, forced us to the lower requirements for their final purity. Thus, we employed gating which was somehow wider from usually employed, and in addition, the instrument internal sort purity control settings were set to allow maximum yield of VSEL on expense of their purity. This was acceptable, since our final goal was not to purify the VSEL, but to enrich the

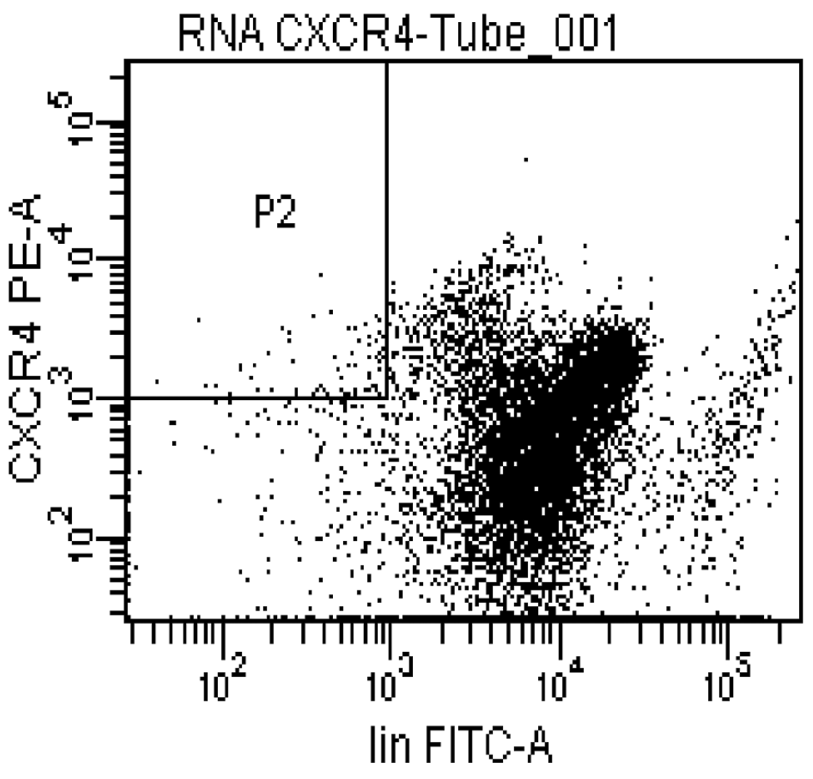

Fig. 3. Events on this dot plot are restricted to those present in the P1 gate on SSC/FSC plot. The position and the extent of the gate P2 were set based on the negative isotype control values, but P2 gate was also designed to sort cells based on subjective judgment.

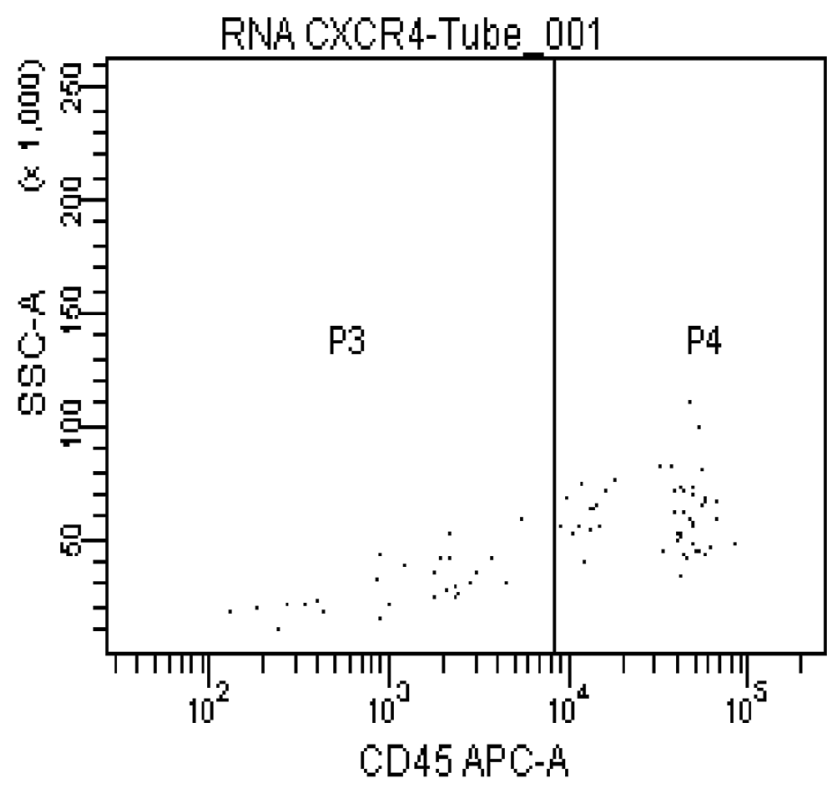

Fig. 4. The events shown on this dot plot were all located within the P2 gate on CXCR4/Lin dot plot. In the gate P4 two subpopulations of CD45 positive cells (bright and dim) are visible. $\mathrm{P} 3$ gate contains VSEL stem cells and it is the gate designed for sorting.

material in these cells. Because there is no specific marker for these cells exclusively available yet, FACS based purification of these cells is very difficult at this point. The high enrichment for VSEL, however, was achieved as confirmed subsequently in the variety of tests performed with these cells [9]. 
Table 1. Selected examples of parameters of UCB samples prepared for FACS sort and the outcome of each individual experiment.

\begin{tabular}{|c|c|c|c|}
\hline Volume of UCB (ml) & MNCs number after lysis & Markers used for sort & Number of sorted cells \\
\hline $40+40$ & $1,5 \times 10^{8}$ & $\mathrm{CXCR}^{+}{ }^{+}$in ${ }^{-} \mathrm{CD} 45^{-}$ & 47000 \\
\hline $60+40$ & $1,5 \times 10^{8}$ & $\mathrm{CXCR}^{+}{ }^{+} \mathrm{in}^{-} \mathrm{CD} 45^{-}$ & 30000 \\
\hline 45 & $0,7 \times 10^{8}$ & $\mathrm{CXCR}^{+}{ }^{+} \mathrm{lin}^{-} \mathrm{CD} 45^{-}$ & 5600 \\
\hline $40+60$ & $3,0 \times 10^{8}$ & $\mathrm{CXCR}^{+}{ }^{1} \mathrm{in}{ }^{-} \mathrm{CD} 45^{-}$ & 22500 \\
\hline $10+10$ & $1,0 \times 10^{8}$ & $\mathrm{CXCR}^{+}{ }^{1} \operatorname{lin}{ }^{-} \mathrm{CD} 45^{-}$ & 60000 \\
\hline 50 & $2,0 \times 10^{8}$ & $\mathrm{CXCR}^{+}{ }^{1} \operatorname{lin} \mathrm{CD} 45^{-}$ & 34000 \\
\hline 55 & $1,1 \times 10^{8}$ & $\mathrm{CD} 4^{+} \operatorname{lin}^{-} \mathrm{CD} 45^{-}$ & 72000 \\
\hline 60 & $1,4 \times 10^{8}$ & $\mathrm{CD}^{2} 33^{+}$lin ${ }^{-} \mathrm{CD} 45^{-}$ & 30500 \\
\hline
\end{tabular}

Another pitfall was to determine the maximum speed that could be safely applied. Although the FACSAria instrument, on which sorts were performed, is designed to enable sorting with the maximum speed of up to 70 thousand events per second, the initial sorts were done at the speed of 8 thousand events per second. Then, after the confirmation that the sorted material is enriched for VSEL, we decided to increase the speed up to $\sim 20$ thousand events per second. We noticed that above this limit, the frequency of cells lost from the sort due to the electronic abort, applied by the instrument to the events which do not meet the number of preset requirements, increased markedly.

The usual sorting time of typical sample of $1-3 \times 10^{8}$ MNCs from UCB was between 0.5 and 3 hours, and depended on the speed of the sorting and the potential sample clotting. The occurrence of the clotting differed from sample to sample and required interruption of the sort and filtering of the cells. Sometimes this had to be repeated several times (up to $\times 4$ ) during one sort.

Although the one stage sorting procedure was performed for most of the times and enabled us to sort large number of VSEL, we also tested two step sorting. In the first step in this latter strategy we sorted lineage negative cells versus CD45 antigen, and next such cells were stained with antibodies against CXCR4, CD34 or CD133 and sorted while expressing these markers. However, it turned out that recovery of VSEL was several fold lower then in one stage sort (the results of tests with two step procedure not shown).

The number of cells sorted from the typical 1-3 $\times 10^{8} \mathrm{MNC}$ sample of UCB, was between few hundred to 50 thousand. It is impossible to established any reliable statistical analyses regarding this parameter, because of the numerous factors including i) the fact that the UCB samples were combined from various deliveries in unknown proportions, ii) the gating and the purity settings were arranged in unrestrictive manner and differed from sort to sort, iii) the speed of the sort varied in individual sorts, and finally, iv) the num- ber of clotting events and related to this filtering of the samples (which may lead to the loss of cells) was also different for each sort. The selected examples of the sorted sample size and the sort outcome are presented in Table 1.

The special attention was paid to the antiseptic conditions of the sort. Although the microbiological purity of the sorted samples was not tested because of the limited size of the samples, cells from the selected sorts were seeded in the culture media and stayed free of bacterial or fungal overgrowth for 1-2 weeks. Another important issue was the viability of sorted cells. The viability tests performed immediately after the sort revealed that $66-82 \%$ of sorted cells were viable, as assessed by tripan blue exclusion test.

In conclusion, we were able to successfully enrich the UCB MNC for the VSEL stem cells and this enrichment was confirmed by PCR for the expression of mRNA for PSC markers as well as by the transmission electron microscope. Further experiments are required though, to improve the sorting conditions for the more efficient enrichment of the UCB for these cells. The identification of VSEL-specific surface markers would be an important step toward this direction. It seems that the possibility of isolation of VSEL from non embryonic tissues is an important step in the adult stem cell research and gives hope for their clinical application in the near future.

\section{References}

[ 1] Loutit JF, Marshall MJ, Nisbet NW, Vaughan JM. Versatile stem cells in bone marrow. Lancet. 1982;2:1090-1093.

[2] Mezey E, Chandross KJ, Harta G, Maki RA, McKercher SR. Turning blood into brain: cells bearing neuronal antigens generated in vivo from bone marrow. Science. 2000;290:17791782 .

[ 3] Orlic D, Kajstura J, Chimenti S, Bodine DM, Leri A, Anversa P. Bone marrow stem cells regenerate infarcted myocardium. Pediatr Transplant. 2003;7(Suppl 3):86-88.

[4] Ratajczak MZ, Kucia M, Reca R, Majka M, Janowska-Wieczorek A, Ratajczak J. Stem cell plasticity revisited: CXCR4- 
positive cells expressing mRNA for early muscle, liver and neural cells 'hide out' in the bone marrow. Leukemia. 2004;18:29-40.

[ 5] Corti S, Locatelli F, Papadimitriou D, Strazzer S, Comi GP. Somatic stem cell research for neural repair: current evidence and emerging perspectives. J Cell Mol Med. 2004;8:329-337.

[6] Shackel N, Jockey D. In pursuit of the "Holy Grail" - stem cells, hepatic injury, fibrogenesis and repair. Hepatology. 2005;41:16-18.

[ 7] Kucia M, Reca R, Jala VR, Dawn B, Ratajczak J, Ratajczak $\mathrm{MZ}$. Bone marrow as a home of heterogenous populations of nonhematopoietic stem cells. Leukemia. 2005;19:1118-1127.

[8] Kucia M, Reca R, Campbell FR et al. A population of very small embryonic-like (VSEL) CXCR $4^{+}$SSEA- $1^{+} \mathrm{OCT} 4^{+}$stem cells identified in adult bone marrow. Leukemia. 2006;20:857-869.

[9] Kucia M, Halasa M, Wysoczynski M. Morphological and molecular characterization of novel population of CXCR4+ SSEA- $4^{+}$Oct- $4^{+}$very small embryonic-like cells purified from human cord blood: preliminary report. Leukemia. 2007;21:297-303.
[ 10]Kucia M, Wysoczynski M, Ratajczak J, Ratajczak MZ. Identification of very small embryonic like (VSEL) stem cells in bone marrow. Cell Tissue Res. 2007;Sep 9:in press.

[11] Kucia M, Zuba-Surma E, Wysoczynski M et al. Physiological and pathological consequences of identification of very small embryonic like (VSEL) stem cells in adult bone marrow. $J$ Physiol Pharmacol. 2006;57 Suppl 5:5-18.

[12] Buzanska L, Machaj EK, Zablocka B, Pojda Z, DomanskaJanik K. Human cord blood-derived cells attain neuronal and glial features in vitro. J Cell Sci. 2002;115:2131-2138.

[13] Baal N, Reisinger K, Jahr H et al. Expression of transcription factor Oct-4 and other embryonic genes in CD133 positive cells from human umbilical cord blood. Thromb Haemost. 2004;92:767-775.

[14] Prat-Vidal C, Roura S, Farre J et al. Umbilical cord bloodderived stem cells spontaneously express cardiomyogenic traits. Transplant Proc. 2007;39:2434-2437.

Submitted: 29 October, 2007 Accepted after reviews: 15 February, 2008 